BMJ Open Sport \& Exercise Medicine

\title{
Estimation of cardiovascular drift through ear temperature during prolonged steady-state cycling: a study protocol
}

\author{
Giovanni Polsinelli (1) , Angelo Rodio 주, Bruno Federico
}

\begin{abstract}
To cite: Polsinelli G, Rodio A Federico $B$. Estimation of cardiovascular drift through ear temperature during prolonged steady-state cycling: a study protocol. BMJ Open Sport \& Exercise Medicine 2021;7:e000907. doi:10.1136/ bmjsem-2020-000907
\end{abstract}

Accepted 8 January 2021
Check for updates

(c) Author(s) (or their employer(s)) 2021. Re-use permitted under CC BY-NC. No commercial re-use. See rights and permissions. Published by BMJ.

Department of Human Sciences, Society and Health, University of Cassino and Southern Lazio, Cassino, Frosinone, Italy

Correspondence to Giovanni Polsinelli; giovanni.polsinelli@unicas.it

\section{ABSTRACT}

Introduction The measurement of heart rate is commonly used to estimate exercise intensity. However, during endurance performance, the relationship between heart rate and oxygen consumption may be compromised by cardiovascular drift. This physiological phenomenon mainly consists of a time-dependent increase in heart rate and decrease in systolic volume and may lead to overestimate absolute exercise intensity in prediction models based on heart rate. Previous research has established that cardiovascular drift is correlated to the increase in core body temperature during prolonged exercise. Therefore, monitoring body temperature during exercise may allow to quantify the increase in heart rate attributable to cardiovascular drift and to improve the estimate of absolute exercise intensity. Monitoring core body temperature during exercise may be invasive or inappropriate, but the external auditory canal is an easily accessible alternative site for temperature measurement. Methods and analysis This study aims to assess the degree of correlation between trends in heart rate and in ear temperature during 120 min of steady-state cycling with intensity of $59 \%$ of heart rate reserve in a thermally neutral indoor environment. Ear temperature will be monitored both at the external auditory canal level with a contact probe and at the tympanic level with a professional infrared thermometer.

Ethics and dissemination The study protocol was approved by an independent ethics committee. The results will be submitted for publication in academic journals and disseminated to stakeholders through summary documents and information meetings.

\section{INTRODUCTION}

The regular practice of cardiorespiratory endurance exercise of appropriate intensity and duration improves aerobic capacity and contributes to maintaining health and physical fitness. ${ }^{1}$ The physiological adaptations responsible for the increase in aerobic capacity mainly depend on the intensity of cardiorespiratory training performed. ${ }^{2}{ }^{3}$ Therefore, an accurate assessment of training intensity is essential to achieve the desired results and avoid overtraining. ${ }^{4}$
Exercise intensity is defined as the amount of metabolic energy required in the unit of time to perform a specific muscular work and can be monitored through variables which are closely correlated to energy expenditure, such as oxygen consumption $\left(\dot{\mathrm{V}} \mathrm{O}_{2}\right)$, power output, speed and heart rate. ${ }^{5}$

In endurance performance, exercise intensity can easily be estimated using a percentage of maximum heart rate (\% HRmax) or a percentage of heart rate reserve $\left(\%\right.$ HRR). ${ }^{1}{ }^{6}$ These methods, based on the assumption of a linear relationship between heart rate and oxygen consumption, were validated in shortterm incremental exercises. ${ }^{7} 8$ However, in prolonged exercises this assumption may be compromised by the physiological phenomenon of cardiovascular drift with potential repercussions on the valid estimation of metabolic intensity. ${ }^{569}$

Cardiovascular drift occurs after 10-15 min of continuous exercise performed at moderate intensity in a neutral or warm environment and consists of a progressive increase in heart rate and a concomitant decrease in systolic volume and mean arterial pressure, without significant changes in cardiac output. ${ }^{10} 11$ These time-dependent changes in cardiovascular responses are associated with a gradual increase in core body temperature ${ }^{12}$ and are exacerbated by dehydration through reduced blood volume and impaired thermoregulatory capacity. ${ }^{13}$

Therefore, monitoring core body temperature during prolonged exercise may allow to quantify the changes in heart rate which are attributable to cardiovascular drift and, consequently, improve the prediction of metabolic intensity. Indeed, during stationary exercise, when the absolute intensity is maintained constant, the 
increase in heart rate may lead to overestimate the metabolic intensity with important implications on the exercise prescription and on the estimate of energy expenditure, in particular in training programmes aimed at weight loss. ${ }^{6}$

The most reliable site for monitoring core body temperature is the pulmonary artery because it transports blood coming directly from the internal districts, while other accepted sites are the oesophagus, rectum and gastrointestinal tract. ${ }^{14}$ Unfortunately, these sites require procedures which are either invasive or inappropriate during sport training. When it is not possible to measure core body temperature directly, alternative easily accessible sites can be used, such as the oral cavity and the external auditory canal. ${ }^{15}$ In clinical settings the sublingual temperature is recommended as a surrogate measure of core body temperature, ${ }^{16}$ but the time required for the thermal stabilisation of this site, the possible interferences deriving from respiration and from the ingestion of liquids or foods discourages its use during and after exercise. ${ }^{1415}$

An alternative site potentially suitable for monitoring body temperature during exercise is the external auditory canal because the tympanic membrane is located near the internal carotid artery and hypothalamic thermoregulatory centres, ${ }^{17}$ although its reliability as a surrogate measure of core body temperature is controversial. $^{16}$

\section{Purpose}

The primary objective of this study is to estimate the extent of the association between trends in heart rate and in ear temperature during prolonged steady-state exercise. This could allow future studies to develop predictive models of absolute intensity based on heart rate and ear temperature, thus indirectly estimating the increase in heart rate attributable to cardiovascular drift.

Because ear temperature can be easily monitored either by measuring the heat transfer by conduction from the external auditory canal with a contact probe or by radiation from the tympanic membrane with an infrared thermometer, the secondary objective is to assess the degree of agreement between the temperature of the external auditory canal and the temperature of the tympanic membrane.

\section{Hypothesis}

During prolonged steady-state exercise in a thermally neutral indoor environment, the increase in heart rate is correlated to the increase in ear temperature.

\section{METHODS}

\section{Subjects}

Thirty healthy and normal weight adult subjects of both sexes will be recruited for the study. The subjects will be trained cyclists with a performance level $\geq 3$ on a $1-5$ scale $^{1819}$ and with medical clearance for competitive cycling.

\section{Inclusion criteria}

1. Subjects of both sexes and aged $18-45$ years.

2. Body mass index (BMI) of $18.5-24.9 \mathrm{~kg} / \mathrm{m}^{2}$.

3. Having received sports medical clearance for competitive cycling in the current year.

4. Cycling training experience $\geq 3$ years for both males and females.

5. Cycling training frequency $\geq 3$ days per week for both males and females.

6. Cycling training time $\geq 5$ hours per week for both males and females.

7. Maximum absolute power output $\geq 320 \mathrm{~W}$ for males and $\geq 235 \mathrm{~W}$ for females.

8. Maximum relative power output $\geq 4.6 \mathrm{~W} / \mathrm{kg}$ for males and $\geq 3.8 \mathrm{~W} / \mathrm{kg}$ for females.

9. Relative $\dot{\mathrm{V}} \mathrm{O}_{2} \max \geq 55 \mathrm{~mL} \cdot \mathrm{min}^{-1} \cdot \mathrm{kg}^{-1}$ for males and $\geq 48 \mathrm{~mL} \cdot \mathrm{min}^{-1} \cdot \mathrm{kg}^{-1}$ for females.

10. Absolute $\dot{\mathrm{VO}_{2}} \max \geq 4.2 \mathrm{~L} / \mathrm{min}$ for males and $\geq 3 \mathrm{~L} /$ min for females.

\section{Exclusion criteria}

1. Presence of cardiovascular diseases.

2. Presence of ear diseases or obstructions in external auditory canal.

3. Presence of diseases capable of influencing thermoregulation.

4. Use of medicines capable of influencing heart rate, blood volume, blood pressure or thermoregulation.

5. Use of medicines capable of influencing psychophysical abilities.

6. Pregnancy or menopause (for females).

\section{Recruitment}

The recruitment of subjects will be carried out in the cities surrounding the University of Cassino and Southern Lazio, in particular at amateur sports associations, gyms, sport events and shops operating in cycling sector through word of mouth, dissemination of announcements and organisation of illustrative meetings. Before participating in study, the experimental protocol will be explained in detail and sufficient time will be allowed to process the information received. Moreover, informed consent and authorisation to process personal data will be acquired in writing and a notification of recruitment will be sent to the subjects' personal doctor to exclude contraindications.

\section{Collection of baseline data}

In the initial phase of the study, participants' personal data will be recorded, copies of an identification document and medical certification for competitive cycling will be deposited. Each participant will be assigned an identification code to guarantee anonymity, inclusion and exclusion criteria will be verified and main anthropometric measurements and familiarisation with experimental procedures will be carried out. In particular, body composition will be estimated from body density ${ }^{20}$ through plicometry by measuring the 
thickness of abdominal, triceps, pectoral, middle axillary, subscapular, suprailiac and quadriceps skin folds. ${ }^{22}{ }^{23}$ The thickness of skin folds will be measured with a professional mechanical plicometer (Skinfold Caliper, GIMA 27320, Metrica S.p.A.) by repeating the operation three times. In addition, with subjects sitting in a comfortable position and after relaxing for least $20 \mathrm{~min}$, the tympanic temperature of both ears will be measured alternately three times with a professional infrared tympanic thermometer (Genius 2, Covidien llc), with the aim of estimating the possible difference between right and left ear. The same measurement procedure will be carried out after isolating the external auditory canal of both ears for 5 min with a preheated antinoise headband (JAZZBAND 2, Moldex-Metric AG \& Co. KG) and repeated again 5 min after removal, with the aim of estimating the possible effect of insulation from external environment on the tympanic temperature. The antinoise headband will be heated by placing it under the subjects' clothes at abdominal level for at least $10 \mathrm{~min}$ before use. To ensure the best hygienic conditions, both earplugs will be coated with a disposable cover obtained from a low-weight ( 3 g) and small size (S) nitrile glove for food use (nyte, walking, Brenta S.r.l. or Reflexx 77, Reflexx S.p.A.).

\section{Cycling tests}

In the central phase of the study, two submaximal cycling tests will be carried out with non-invasive monitoring of heart rate, external auditory canal temperature and tympanic temperature. The tests will be spaced from a minimum of 24 hours up to a maximum of 10 days. In female athletes, cycling tests will not be performed during the mid-luteal phase of the menstrual cycle to limit the influence on body temperature and heart rate. ${ }^{24}$

Before performing the cycling tests, the baseline values of heart rate and external auditory canal temperature will be monitored for $10 \mathrm{~min}$ with subjects sitting in a comfortable position and after relaxing for at least $20 \mathrm{~min}$. These baseline values will be obtained by averaging the last $5 \mathrm{~min}$ of monitoring. The baseline value of the tympanic temperature will be determined by carrying out a measurement at the end of the monitoring of the other physiological variables to avoid influences on the heart rate.

The first cycling test will consist of incremental exercise up to $80 \%$ of the theoretical maximum heart rate (HRmax) estimated with the formula 220 - age..$^{25}$ This test will be necessary to estimate the subject-specific relationship between heart rate and power output, that will be used to determine the power corresponding to the target intensity of the second cycling test. The choice of this submaximal test instead of a maximal test may limit the estimation of the exercise intensity. However, the use of a maximal incremental test with respiratory gas monitoring would require more resources and adequate clinical care limiting the feasibility of the study in a nonhospital setting.

Incremental cycling test protocol:
1. Initial intensity of $100 \mathrm{~W}$ for males and $50 \mathrm{~W}$ for females at 70-90 rpm and increments of $25 \mathrm{~W}$ every 3 min until achieving and maintaining $80 \%$ HRmax for $3 \mathrm{~min}$.

2. $10 \mathrm{~min}$ cool down with intensity of $50-100 \mathrm{~W}$ at $70-90 \mathrm{rpm}$.

The second cycling test will consist of a prolonged steady-state exercise at the upper limit of moderate intensity in a thermally neutral indoor environment, with the purpose of assessing the association between trends in heart rate and in ear temperature.

The moderate exercise intensity of the prolonged steady-state cycling test will be estimated using a percentage of heart rate reserve. ${ }^{1626-28}$ In cardiorespiratory endurance exercise, a moderate level of intensity is between $40 \%$ and $59 \%$ of heart rate reserve or oxygen consumption reserve. ${ }^{1}$ The target heart rate (HRtarget) corresponding to the desired exercise intensity will be calculated using the following equation:

$$
\text { HRtarget }=\{[(\text { HRmax }- \text { HRrest }) \times \% \text { HRR }] / 100\}+\text { HRrest }
$$

where HRmax is the maximum heart rate, HRrest the resting heart rate and \% HRR the percentage of heart rate reserve. The target heart rate will be converted into power using the subject-specific relationship between heart rate and power output, in order to maintain the absolute exercise intensity constant.

Prolonged steady-state cycling test protocol:

1. 10 min warm up with increasing intensity until reaching the target ( $5 \mathrm{~min}$ at $50 \%$ of target, $3 \mathrm{~min}$ at $80 \%$ of target and $2 \mathrm{~min}$ at $90 \%$ of target) at $70-90 \mathrm{rpm}$.

2. 120 min exercise with intensity equal to the power corresponding to $59 \%$ HRR at $70-90 \mathrm{rpm}$.

3. 10 min cool down with intensity equal to $50 \%$ of target at $70-90 \mathrm{rpm}$.

The cycling tests will be carried out with one of the bicycles usually used by athletes to maximise comfort during the exercise and wearing a light cycling uniform. Bicycles will be mounted (fixing the rear carriage) on a professional training roller with magnetic brake with 30 certified positions (MagneticDays JARVIS, O.R.F. S.r.l.) equipped with software (MD Training, O.R.F. S.r.l.) to develop training protocols, to acquire and transfer data and to view the working parameters in real time (elapsed time, speed, power output, pedalling cadence and heart rate). This professional training roller used for the cycling tests is capable of automatically adjusting the resistance based on the pedalling rate to maintain the power output constant.

During the cycling tests, heart rate, external auditory canal temperature of the right ear and tympanic temperature (infrared radiation) of the left ear will be monitored. Before carrying out temperature measurements, the external auditory canal of both ears will be visually inspected to verify the absence of obstructions and/or anomalies. 
Heart rate will be monitored with a wireless chest strap heart rate monitor (Soft Strap Premium Heart Rate Monitor, Garmin Ltd) positioned under the chest. For data transfer, the heart rate monitor will be associated with the training roller through a short-range wireless connection (ant + ).

The external auditory canal temperature will be monitored in the right ear through continuous measurement with a wearable device (C-Temp, Cosinuss $\mathrm{GmbH}$ ) consisting of a retro-ear module and an adjustable contact probe. A firmware modification was made by the manufacturer to obtain absolute temperature values without corrections. For data transfer, the auricular device will be associated with a smartphone or tablet through a shortrange wireless connection (Bluetooth). The software application (cosinuss $^{\circ}$ saveXport, Cosinuss $\mathrm{GmbH}$ or cosinuss $^{\circ} \mathrm{Lab}$, Cosinuss $\mathrm{GmbH}$ ) provided by the manufacturer allows to view the measured temperature in real time and to acquire data for subsequent transfer to a PC. To ensure the best hygienic conditions, both the ear probe and the retro-ear module will be coated with a disposable cover obtained from a low-weight $(\sim 3 \mathrm{~g})$ and small size (S) nitrile glove for food use (nyte, walking, Brenta S.r.l. or Reflexx 77, Reflexx S.p.A.).

To avoid the release of the probe from external auditory canal or unwanted movements, a suitably modified antinoise headband (JAZZ-BAND 2, Moldex-Metric AG \& Co. KG) will be used. The modification consists in the partial removal of the support of the right earplug and the insertion on the remaining part of a small cylinder in non-toxic plasticised polyvinyl chloride able to accommodate the base of the probe and exert a slight pressure $(\sim 1.2 \mathrm{~N})$. The unmodified contralateral earplug will be positioned at the entrance of the left external auditory canal. To ensure the best hygienic conditions, a disposable nitrile cover will be applied to both earplugs, as in the case of the auricular device. In addition, to adjust the length of the probe and redistribute the pressure exerted by the antinoise headband, disposable cotton pads will be inserted between the probe and the entrance of the external auditory canal.

The tympanic temperature will be monitored in the left ear by manual sampling with a professional infrared tympanic thermometer (Genius 2, Covidien llc) by carrying out the otoscopic manoeuver to straighten the external auditory canal and directing the probe towards the tympanic membrane. This manoeuver, which will be carried out by a trained operator, allows for more accurate tympanic temperature measurements and consists of moderate traction posteriorly and superiorly of the auricle carried out in the midpoint between the apex of the helix and the lower end of the lobule. ${ }^{29}$ The tympanic thermometer will be set in ear mode (EAR) to obtain absolute temperature measurements without corrections for equivalence with other sites. This instrument is equipped with replaceable probe covers (Tympanic Probe Covers, 303030, Covidien llc) to ensure the best hygienic conditions.
During the incremental cycling test, the tympanic temperature will be measured every $3 \mathrm{~min}$, while in the prolonged cycling test every $5 \mathrm{~min}$. Before carrying out these measurements, the left earplug of the antinoise headband will be temporarily moved to allow access to the external auditory canal.

In order to limit dehydration (body weight loss $>2 \%$ ) during prolonged steady-state test, subjects will take every $10 \mathrm{~min}$ a dose of mineral water (SANGEMINI, Acque Minerali d'Italia S.p.A.) at room temperature for a total quantity between 0.4 and $0.8 \mathrm{~L} /$ hour, based on the initial body weight. ${ }^{30}$

Hydration based on athletes' body weight:

1. $50-<60 \mathrm{~kg}=0.4 \mathrm{~L} /$ hour.

2. $60-<70 \mathrm{~kg}=0.5 \mathrm{~L} /$ hour.

3. $70-<80 \mathrm{~kg}=0.6 \mathrm{~L} /$ hour.

4. $80-<90 \mathrm{~kg}=0.7 \mathrm{~L} /$ hour.

5. $\geq 90 \mathrm{~kg}=0.8 \mathrm{~L} /$ hour.

In order to prevent disorders or discomfort due to permanence on the bicycle during prolonged steadystate test, subjects will perform an out of the saddle every 20 min lasting $\sim 5 \mathrm{~s}$.

Body weight of subjects will be measured before and after the cycling tests with a digital scale (RD-953, innerScan DUAL, TANITA Corporation) to assess dehydration. The measurements will be carried out with the body dry in undergarments and possibly after urinating.

Before the cycling tests, study participants should:

1. Take at least $1.9 \mathrm{~L} /$ day of water in the previous 48 hours.

2. Take an adequate amount of carbohydrates $(\sim 60 \%$ of $\mathrm{kcal} /$ day) in the previous 48 hours.

3. Refrain from taking caffeine, alcohol, nicotine or other stimulants for at least 4 hours.

4. Take the last meal 4-6hours before.

5. Refrain from moderate physical activity for at least 2 hours and from strenuous exercise for at least 14 hours.

6. Take $500 \mathrm{~mL}$ of mineral water in the previous $60 \mathrm{~min}$.

7. Rest for $\geq 20 \mathrm{~min}$ before monitoring the baseline values of the physiological variables.

During this phase of the study, subjects will draw up a diary on the diet and exercise for the 48 hours preceding the cycling tests to assess the congruence with the indications provided.

At the end of the cycling tests, participants will receive packaged food and beverages and a self-compiled questionnaire to investigate the perceived level of comfort/ discomfort during the experimental procedures. The questionnaire is composed of the following four items, each based on a 5-point Likert scale: the use of the chest strap heart rate monitor, the auricular device supported by the modified antinoise headband, the tympanic thermometer and the training roller.

\section{Statistical analyses}

Numerical data (age, height, weight, BMI, percentage of body fat, HRrest, theoretical HRmax and power output 
at $80 \%$ HRmax) will be presented using mean and SD in the case of normal distributions or median and IQR in the case of non-normal distributions, while categorical data (sex and performance level) will be presented through frequency tables.

The degree of agreement between the two measurements of ear temperature (ie, external auditory canal temperature of the right ear and tympanic temperature of the left ear) will be assessed through the Bland-Altman analysis. ${ }^{31}$ The association between two time series of numeric data (ie, heart rate vs external auditory canal temperature and heart rate vs tympanic temperature) will be assessed through the analysis of cross correlation. $^{32}$

Statistical significance will be set at a level of $\mathrm{p}<0.05$.

\section{Work environment}

The study will be conducted in a suitable room equipped with sanitary facilities at the Laboratory of Physiology of exercise and sport 'Marco Marchetti' of the Department of Human Sciences, Society and Health of the University of Cassino and Southern Lazio. In this room there will be an adequate air exchange between inside and outside, a temperature of $20^{\circ} \mathrm{C}-25^{\circ} \mathrm{C}$ and a relative humidity of $40 \%-65 \%$. These environmental conditions are comfortable for carrying out cardiorespiratory exercise with a light cycling uniform. During the experimental procedures, the temperature and relative humidity will be monitored with a thermo-hygrometer with data logger and display for real-time visualisation of the measured values (PCE-THB 40, PCE Deutschland $\mathrm{GmbH})$.

\section{Safety of procedures}

The study was approved by an independent ethics committee and will be conducted in compliance with the European Union's Good Clinical Practice standards and in accordance with the Declaration of Helsinki. The experimental procedures will be carried out by qualified sports science experts and a medical doctor.

To ensure adequate safety standards, the room destined for the experimentation will be equipped with a first aid kit for the cardio-pulmonary resuscitation including an external semiautomatic defibrillator, an Ambu resuscitation flask for adults with mask, an assortment of sterile Guedel cannulas, a tongue forceps and a conical helical mouth opener.

Specific containment measures will be taken to prevent the risk of contagion of infectious diseases, with special reference to SARS-CoV-2. The laboratory intended for the experimentation will be sanitised periodically, while the support surfaces and the instruments will be thoroughly disinfected before and after use. In addition, a continuous air exchange between inside and outside will be guaranteed. The operators involved in the study will wash their hands thoroughly and wear disposable masks (FFP2) and gloves.

\section{Personal data processing}

The personal data of the study participants will be processed anonymously and exclusively for the realisation of the study and the purposes of scientific research, in accordance with national legislation (Legislative Decree 196/2003 and subsequent adaptation of Legislative Decree 101/2018) and the provisions of the European regulation (GDPR 679/2016) on the protection of personal data.

\section{Patient and public involvement}

Representatives of cycling organisations worked with the investigators to refine the experimental protocol; they will also be actively involved in the recruitment of cyclists. At the end of the study, a dissemination document will be delivered to the participants and the interested public to illustrate the results and potential practical applications of this research. In addition, information meetings will be held in academic and sport contexts.

Acknowledgements The authors would like to thank the representatives of the cycling organisations for the contribution provided to refine the experimental protocol and the support for the recruitment of cyclists.

Contributors GP designed the study as part of a PhD degree. GP and AR conceived the research project. BF provided assistance with the study design and analysis methods. All authors provided intellectual contributions in further designing the study for each of their respective areas of expertise. GP drafted the manuscript, with feedback provided by all authors. All authors approved the final manuscript.

Funding This work was conducted as part of GP's PhD studies.

Competing interests None declared.

Patient consent for publication Not required.

Ethics approval The study protocol was approved by the 'Lazio 2' Ethics Committee with Protocol No. 0219118 of 11.12.2019.

Provenance and peer review Not commissioned; externally peer reviewed.

Data availability statement Data sharing not applicable as no datasets were generated and/or analysed for this study.

Open access This is an open access article distributed in accordance with the Creative Commons Attribution Non Commercial (CC BY-NC 4.0) license, which permits others to distribute, remix, adapt, build upon this work non-commercially, and license their derivative works on different terms, provided the original work is properly cited, appropriate credit is given, any changes made indicated, and the use is non-commercial. See: http://creativecommons.org/licenses/by-nc/4.0/.

\section{ORCID iDs}

Giovanni Polsinelli http://orcid.org/0000-0002-9484-9720

Angelo Rodio http://orcid.org/0000-0003-2964-7193

Bruno Federico http://orcid.org/0000-0002-8629-4366

\section{REFERENCES}

1 Garber CE, Blissmer B, Deschenes MR, et al. American College of Sports Medicine position stand. Quantity and quality of exercise for developing and maintaining cardiorespiratory, musculoskeletal, and neuromotor fitness in apparently healthy adults: guidance for prescribing exercise. Med Sci Sports Exerc 2011;43:1334-59.

2 Gormley SE, Swain DP, High R, et al. Effect of intensity of aerobic training on VO2max. Med Sci Sports Exerc 2008;40:1336-43.

3 Swain DP, Franklin BA. VO2 reserve and the minimal intensity for improving cardiorespiratory fitness. Med Sci Sports Exerc 2002;34:152-7.

4 Kuipers $\mathrm{H}$, Keizer HA. Overtraining in elite athletes. Review and directions for the future. Sports Med 1988;6:79-92.

5 Jeukendrup A, VanDiemen A. Heart rate monitoring during training and competition in cyclists. J Sports Sci 1998;16 Suppl:91-9. 
6 da Cunha FA, Farinatti PdeTV, Midgley AW. Methodological and practical application issues in exercise prescription using the heart rate reserve and oxygen uptake reserve methods. J Sci Med Sport 2011:14:46-57.

7 Davis JA, Convertino VA. A comparison of heart rate methods for predicting endurance training intensity. Med Sci Sports 1975;7:295-8.

8 Swain DP, Leutholtz BC. Heart rate reserve is equivalent to \%VO2 reserve, not to \%VO2max. Med Sci Sports Exerc 1997;29:410-4.

9 Vogt S, Heinrich L, Schumacher YO, et al. Power output during stage racing in professional road cycling. Med Sci Sports Exerc 2006;38:147-51.

10 Ekelund LG. Circulatory and respiratory adaptation during prolonged exercise of moderate intensity in the sitting position. Acta Physiol Scand 1967;69:327-40.

11 Rowell LB. Human cardiovascular adjustments to exercise and thermal stress. Physiol Rev 1974;54:75-159.

12 Fritzsche RG, Switzer TW, Hodgkinson BJ, et al. Stroke volume decline during prolonged exercise is influenced by the increase in heart rate. J Appl Physiol 1999;86:799-805.

13 Coyle EF, González-Alonso J. Cardiovascular drift during prolonged exercise: new perspectives. Exerc Sport Sci Rev 2001;29:88-92.

14 Moran DS, Mendal L. Core temperature measurement: methods and current insights. Sports Med 2002;32:879-85.

15 Lim CL, Byrne C, Lee JK. Human thermoregulation and measurement of body temperature in exercise and clinical settings. Ann Acad Med Singap 2008;37:347-53.

16 Hooper VD, Andrews JO. Accuracy of noninvasive core temperature measurement in acutely ill adults: the state of the science. Biol Res Nurs 2006;8:24-34.

17 Yeoh WK, Lee JKW, Lim HY, et al. Re-visiting the tympanic membrane vicinity as core body temperature measurement site. PLoS One 2017;12:e0174120.

18 Decroix L, De Pauw K, Foster C, et al. Guidelines to classify female subject groups in Sport-Science research. Int J Sports Physiol Perform 2016;11:204-13.
19 De Pauw K, Roelands B, Cheung SS, et al. Guidelines to classify subject groups in sport-science research. Int J Sports Physiol Perform 2013;8:111-22.

20 Heyward VH, Wagner DR. Applied body composition assessment. In: Human kinetics. 2nd edn, 2004: 280.

21 Siri WE. Body composition from fluid spaces and density: analysis of methods. 1961. Nutrition 1993;9:480-92.

22 Jackson AS, Pollock ML. Practical assessment of body composition. Phys Sportsmed 1985;13:76-90.

23 Pollack ML, Schmidt DH, Jackson AS. Measurement of cardiorespiratory fitness and body composition in the clinical setting. Compr Ther 1980;6:12-27.

24 Janse de Jonge XAK. Effects of the menstrual cycle on exercise performance. Sports Med 2003;33:833-51.

25 Fox SM, Naughton JP, Haskell WL. Physical activity and the prevention of coronary heart disease. Ann Clin Res 1971;3:404-32.

26 Karvonen MJ, Kentala E, Mustala O. The effects of training on heart rate; a longitudinal study. Ann Med Exp Biol Fenn 1957;35:307-15.

27 Lounana J, Campion F, Noakes TD, et al. Relationship between \%HRmax, \%HR reserve, \%VO2max, and \%VO2 reserve in elite cyclists. Med Sci Sports Exerc 2007;39:350-7.

28 Earhart EL, Weiss EP, Rahman R, et al. Effects of oral sodium supplementation on indices of thermoregulation in trained, endurance athletes. J Sports Sci Med 2015;14:172-8.

29 Terndrup TE, Rajk J. Impact of operator technique and device on infrared emission detection tympanic thermometry. J Emerg Med 1992;10:683-7.

30 American College of Sports Medicine, Sawka MN, Burke LM, et al. American College of Sports Medicine position stand. Exercise and fluid replacement. Med Sci Sports Exerc 2007;39:377-90.

31 Bland JM, Altman DG. Statistical methods for assessing agreement between two methods of clinical measurement. Lancet 1986;1:307-10.

32 Nelson-Wong E, Howarth S, Winter DA, et al. Application of autocorrelation and cross-correlation analyses in human movement and rehabilitation research. $J$ Orthop Sports Phys Ther 2009;39:287-95. 